\title{
Correction to: Keyword Search Based Mashup Construction with Guaranteed Diversity
}

Huanyu Cheng, Ming Zhong, Jian Wang, and Tieyun Qian

\section{Correction to:}

Chapter "Keyword Search Based Mashup Construction with Guaranteed Diversity" in: S. Hartmann et al. (Eds.): Database and Expert Systems Applications, LNCS 11707, https://doi.org/10.1007/978-3-030-27618-8_31

In the originally published version of chapter 31 the funding information in the acknowledgement section was incomplete. This has now been corrected. 